\title{
Efficient deconvolution methods for astronomical imaging: algorithms and IDL-GPU codes
}

\author{
M. Prato ${ }^{1}$, R. Cavicchioli ${ }^{1}$, L. Zanni ${ }^{1}$, P. Boccacci ${ }^{2}$, and M. Bertero ${ }^{2}$ \\ 1 Dipartimento di Matematica Pura ed Applicata, Università di Modena e Reggio Emilia, via Campi 213/b, 41125 Modena, Italy
e-mail: marco.prato@unimore.it \\ 2 Dipartimento di Informatica e Scienze dell’Informazione, Università di Genova, via Dodecaneso 35, 16146 Genova, Italy \\ Received 19 December 2011 / Accepted 16 January 2012
}

\section{ABSTRACT}

\begin{abstract}
Context. The Richardson-Lucy method is the most popular deconvolution method in astronomy because it preserves the number of counts and the non-negativity of the original object. Regularization is, in general, obtained by an early stopping of Richardson-Lucy iterations. In the case of point-wise objects such as binaries or open star clusters, iterations can be pushed to convergence. However, it is well-known that Richardson-Lucy is an inefficient method. In most cases and, in particular, for low noise levels, acceptable solutions are obtained at the cost of hundreds or thousands of iterations, thus several approaches to accelerating Richardson-Lucy have been proposed. They are mainly based on Richardson-Lucy being a scaled gradient method for the minimization of the Kullback-Leibler divergence, or Csiszár I-divergence, which represents the data-fidelity function in the case of Poisson noise. In this framework, a line search along the descent direction is considered for reducing the number of iterations.

Aims. A general optimization method, referred to as the scaled gradient projection method, has been proposed for the constrained minimization of continuously differentiable convex functions. It is applicable to the non-negative minimization of the Kullback-Leibler divergence. If the scaling suggested by Richardson-Lucy is used in this method, then it provides a considerable increase in the efficiency of Richardson-Lucy. Therefore the aim of this paper is to apply the scaled gradient projection method to a number of imaging problems in astronomy such as single image deconvolution, multiple image deconvolution, and boundary effect correction.

Methods. Deconvolution methods are proposed by applying the scaled gradient projection method to the minimization of the Kullback-Leibler divergence for the imaging problems mentioned above and the corresponding algorithms are derived and implemented in interactive data language. For all the algorithms, several stopping rules are introduced, including one based on a recently proposed discrepancy principle for Poisson data. To attempt to achieve a further increase in efficiency, we also consider an implementation on graphic processing units.

Results. The proposed algorithms are tested on simulated images. The acceleration of scaled gradient projection methods achieved with respect to the corresponding Richardson-Lucy methods strongly depends on both the problem and the specific object to be reconstructed, and in our simulations the improvement achieved ranges from about a factor of 4 to more than 30. Moreover, significant accelerations of up to two orders of magnitude have been observed between the serial and parallel implementations of the algorithms. The codes are available upon request.
\end{abstract}

Key words. methods: numerical - methods: data analysis - techniques: image processing

\section{Introduction}

The Richardson-Lucy (RL) algorithm (Richardson 1972; Lucy 1974) is a renowned iterative method for image deconvolution in astronomy and other sciences. Here, we define $\boldsymbol{g}$ to be the detected image and $A$ the imaging matrix given by $A \boldsymbol{f}=\boldsymbol{K} *$ $\boldsymbol{f}$, where $\boldsymbol{K}$ is the point spread function (PSF) and $*$ denotes a convolution. If the PSF is then normalized to unit volume, the iteration, as modified by Snyder (Snyder 1990), is

$\boldsymbol{f}^{(k+1)}=\boldsymbol{f}^{(k)} \circ A^{\mathrm{T}} \frac{\boldsymbol{g}}{A \boldsymbol{f}^{(k)}+\boldsymbol{b}}$,

where $A^{\mathrm{T}}$ is the transposed matrix, $\boldsymbol{b}$ is a known array representing background emission, $\boldsymbol{x} \circ \boldsymbol{y}$ denotes the pixel by pixel product of two equally-sized arrays $\boldsymbol{x}, \boldsymbol{y}$, and $\boldsymbol{x} / \boldsymbol{y}$ their quotient.

It is well-known that the method has several interesting features. The result of each iteration is non-negative and robust against small errors in the PSF, and that flux is conserved both globally and locally if $\boldsymbol{b}=0$.

In such a case, it has also been proven by several authors (see, for instance, Natterer \& Wübbeling 2001) that the iterations converge to either a maximum likelihood solution for Poisson data (Shepp \& Vardi 1982) or, equivalently, to a minimizer of the Kullback-Leibler (KL) divergence, which is also known as the Csiszár I-divergence (Csiszár 1991), given by

$$
\begin{array}{r}
J_{0}(\boldsymbol{f} ; \boldsymbol{g})=\sum_{\mathbf{m} \in S}\left\{\boldsymbol{g}(\mathbf{m}) \ln \frac{\boldsymbol{g}(\mathbf{m})}{(A \boldsymbol{f})(\mathbf{m})+\boldsymbol{b}(\mathbf{m})}\right. \\
+(A \boldsymbol{f})(\mathbf{m})+\boldsymbol{b}(\mathbf{m})-\boldsymbol{g}(\mathbf{m})\},
\end{array}
$$

where $S$ is the set of values of the multi-index $\mathbf{m}$ labeling the image pixels.

As shown in Barrett \& Meyers (2003), the non-negative minimizers of $J_{0}(\boldsymbol{f} ; \boldsymbol{g})$ are sparse objects, i.e. they consist of bright spots over a black background. Therefore, in the case of simple astronomical objects, such as binaries or open star clusters, the algorithm can be pushed to convergence (examples are given in Sect. 4), while, in the case of more complex objects, an early stopping of the iterations, providing a "regularization effect", is required. The problem of introducing suitable stopping rules is briefly discussed in Sect. 3. 
The main disadvantage of the RL algorithm is that it is not very efficient: it may require hundreds or thousands of iterations for images with a large number of counts (low Poisson noise). In the case of large-scale images or multiple images of the same target, the computational cost can become prohibitive. For this reason, several acceleration schemes have been proposed, of which we mention a few.

The first is the "multiplicative relaxation" proposed by Llacer \& Núñez (1990), which consists in replacing the iteration of Eq. (1) by

$\boldsymbol{f}^{(k+1)}=\boldsymbol{f}^{(k)} \circ\left(A^{\mathrm{T}} \frac{\boldsymbol{g}}{A \boldsymbol{f}^{(k)}+\boldsymbol{b}}\right)^{\alpha}$

with $\alpha>1$. Convergence is proved in Iusem (1991) for $\alpha<$ 2. As demonstrated in Lantéri et al (2001), this approach can provide a reduction in the number of iterations by a factor of $\alpha$, with essentially the same cost per iteration. For low numbers of counts numerical convergence has been found also for $\alpha>2$ (Anconelli et al. 2005). A "linear relaxation" is investigated in Adorf et al. (1992). It can be written in the form

$\boldsymbol{f}^{(k+1)}=\boldsymbol{f}^{(k)}-\lambda_{k} \boldsymbol{f}^{(k)} \circ\left(\mathbf{1}-A^{\mathrm{T}} \frac{\boldsymbol{g}}{A \boldsymbol{f}^{(k)}+\boldsymbol{b}}\right)$,

where $\lambda_{k}>1$ (for $\lambda_{k}=1$ the RL algorithm is re-obtained) and $\mathbf{1}$ is the array with all entries equal to 1 . Since the quantity in brackets is the gradient of $J_{0}(\boldsymbol{f} ; \boldsymbol{g})$, we note that RL is a scaled gradient method with a scaling given by $\boldsymbol{f}^{(k)}$ at iteration $k$, and that the relaxation method is essentially a line search along this descent direction, which can be performed by minimizing the objective function $J_{0}(\boldsymbol{f} ; \boldsymbol{g})$ (Adorf et al. 1992) or applying the Armijo rule (Lantéri et al. 2001). A moderate increase in efficiency is then observed by these authors. The values reached after convergence of the algorithms can be inferred from general results of optimization theory (Bertsekas 2003). Finally, a greater increase in efficiency on the order of ten, is observed using an acceleration method proposed by Biggs \& Andrews (1997), which exploits a suitable extrapolation along the trajectory of the iterates, and is implemented in the deconvlucy function of the Image Processing MATLAB toolbox. The problem with this method is that no convergence proof is available and, in our experience, a deviation from the trajectory of RL iterations is sometimes observed, providing unreliable results.

Bonettini et al. (2009) developed an optimization method, which they called scaled gradient projection (SGP) method, to constrain the minimization of a convex function, and proved that its convergence occurs under mild conditions. This method can be quite naturally applied to the non-negative minimization of the KL divergence, using the scaling of the gradient suggested by RL, hence this application of SGP can also be considered as a more efficient version of RL. In Bonettini et al. (2009), the performance of the new method is compared with that of RL and the Biggs \& Andrews method, as implemented in MATLAB, providing an improvement in efficiency comparable to that of the latter method, but sometimes better and without its drawbacks. Further applications of SGP in image restoration problems can be found e.g. in Benvenuto et al. (2010), Bonettini \& Prato (2010), and Zanella et al. (2009).

The purpose of this paper is not only to illustrate the features of SGP to the astronomical community, but also to extend its application to the problems of multiple image deconvolution and boundary effect correction. The first problem is fundamental, for instance, to the reconstruction of the images of the future interferometer of the Large Binocular Telescope (LBT), denoted LINC-NIRVANA (Herbst et al. 2003), while the second problem is important in both single and multiple image deconvolution. All the algorithms are implemented in interactive data language (IDL) and the codes will be freely distributed. Moreover, we present an implementation for graphic processor unit (GPU) is also provided. In this paper, we consider only the constraint of non-negativity. Bonettini et al. (2009) investigated both non-negativity and flux conservation and provided an efficient algorithm, for computing the projection on the convex set defined by the constraints. However, their numerical experiments seem to demonstrate that the additional flux constraint does not significantly improve the reconstructions.

The paper is organized as follows. In Sect. 2, after a brief description of the general SGP algorithm in the case of nonnegativity constraint, we derive its application to the problems of both single and multiple image deconvolution and boundary effect correction. In Sect. 3, we describe the IDL and GPU codes and in Sect. 4 we discuss our numerical experiments illustrating the increase in efficiency achievable with the proposed methods. In Sect. 5, we discuss possible implementation improvements and extensions to regularized problems.

\section{The deconvolution methods}

We first describe the monotone SGP algorithm for minimizing a convex and differentiable function on the non-negative orthant. For the general version of the algorithm including a flux constraint, we refer to Bonettini et al. (2009). Next, we outline the application of SGP to the three imaging problems mentioned in the Introduction.

\subsection{The scaled gradient projection (SGP) method}

The SGP scheme is a gradient method for the solution of the problem

$\min _{f \geq 0} J_{0}(\boldsymbol{f} ; \boldsymbol{g})$,

where $J_{0}(\boldsymbol{f} ; \boldsymbol{g})$ is a convex and continuously differentiable function defined for each one of the problems considered in this paper. Each SGP iteration is based on the descent direction $\boldsymbol{d}^{(k)}=\boldsymbol{y}^{(k)}-\boldsymbol{f}^{(k)}$, where

$\boldsymbol{y}^{(k)}=P_{+}\left(\boldsymbol{f}^{(k)}-\alpha_{k} D_{k} \nabla J_{0}\left(\boldsymbol{f}^{(k)} ; \boldsymbol{g}\right)\right)$

is defined by combining a scaled steepest descent direction with a projection on the non-negative orthant. The matrix $D_{k}$ in Eq. (6) is chosen in the set $\mathcal{D}$ of the $n \times n$ diagonal positive definite matrices, whose diagonal elements have values between $L_{1}$ and $L_{2}$ for given thresholds $0<L_{1}<L_{2}$.

The main SGP steps are given in algorithm 1. The global convergence of the algorithm is obtained by means of the standard monotone Armijo rule in the line-search procedure described in step 5 (see Bonettini et al. 2009).

We emphasize that any choice of the steplength $\alpha_{k} \in$ [ $\left.\alpha_{\min }, \alpha_{\max }\right]$ and the scaling matrix $D_{k} \in \mathcal{D}$ are allowed; this freedom of choice can then be fruitfully exploited for introducing performance improvements.

An effective selection strategy for the steplength parameter is obtained by adapting to the context of the scaling gradient methods the Barzilai \& Borwein (1988) rules (hereafter denoted BB), which are widely used in standard nonscaled gradient methods. When the scaled direction $D_{k} \nabla J_{0}\left(\boldsymbol{f}^{(k)} ; \boldsymbol{g}\right)$ is exploited within a 


\begin{tabular}{l}
$\overline{\text { Algorithm } 1 \text { Scaled gradient projection (SGP) method }}$ \\
\hline Choose the starting point $\boldsymbol{f}^{(0)} \geq 0$ and set the parameters $\beta, \theta \in(0,1)$ \\
$0<\alpha_{\min }<\alpha_{\max }$.
\end{tabular}

For $k=0,1,2, \ldots$ DO THE FOLLOWING STEPS:

STEP 1. Choose the parameter $\alpha_{k} \in\left[\alpha_{\min }, \alpha_{\max }\right]$ and the scaling matrix $D_{k} \in \mathcal{D}$;

SteP 2. Projection:

$$
\boldsymbol{y}^{(k)}=P_{+}\left(\boldsymbol{f}^{(k)}-\alpha_{k} D_{k} \nabla J_{0}\left(\boldsymbol{f}^{(k)} ; \boldsymbol{g}\right)\right) ;
$$

STEP 3. Descent direction: $\boldsymbol{d}^{(k)}=\boldsymbol{y}^{(k)}-\boldsymbol{f}^{(k)}$;

STEP 4. Set $\lambda_{k}=1$;

STEP 5. Backtracking loop:

$$
\begin{aligned}
& \text { let } J_{\text {new }}=J_{0}\left(\boldsymbol{f}^{(k)}+\lambda_{k} \boldsymbol{d}^{(k)} ; \boldsymbol{g}\right) ; \\
& \text { IF } \quad J_{\text {new }} \leq J_{0}\left(\boldsymbol{f}^{(k)} ; \boldsymbol{g}\right)+\beta \lambda_{k} \nabla J_{0}\left(\boldsymbol{f}^{(k)} ; \boldsymbol{g}\right)^{T} \boldsymbol{d}^{(k)} \\
& \text { THEN } \\
& \quad \text { go to step 6; } \\
& \text { ELSE } \\
& \quad \text { set } \lambda_{k}=\theta \lambda_{k} \text { and go to step 5. } \\
& \text { ENDIF }
\end{aligned}
$$

STEP 6. Set $\boldsymbol{f}^{(k+1)}=\boldsymbol{f}^{(k)}+\lambda_{k} \boldsymbol{d}^{(k)}$.

$\underline{\text { END }}$

step of the form $\left(\boldsymbol{f}^{(k)}-\alpha_{k} D_{k} \nabla J_{0}\left(\boldsymbol{f}^{(k)} ; \boldsymbol{g}\right)\right)$, the BB steplength rules become

$$
\begin{aligned}
& \alpha_{k}^{(B B 1)}=\frac{\boldsymbol{s}^{(k-1)^{T}} D_{k}^{-1} D_{k}^{-1} \boldsymbol{s}^{(k-1)}}{\boldsymbol{s}^{(k-1)^{T}} D_{k}^{-1} \boldsymbol{z}^{(k-1)}}, \\
& \alpha_{k}^{(B B 2)}=\frac{\boldsymbol{s}^{(k-1)^{T}} D_{k} \boldsymbol{z}^{(k-1)}}{\boldsymbol{z}^{(k-1)^{T}} D_{k} D_{k} \boldsymbol{z}^{(k-1)}},
\end{aligned}
$$

where $\boldsymbol{s}^{(k-1)}=\boldsymbol{f}^{(k)}-\boldsymbol{f}^{(k-1)}$ and $\boldsymbol{z}^{(k-1)}=\nabla J_{0}\left(\boldsymbol{f}^{(k)} ; \boldsymbol{g}\right)-\nabla J_{0}\left(\boldsymbol{f}^{(k-1)} ; \boldsymbol{g}\right)$. In SGP, we constrain the values produced by these rules into the interval $\left[\alpha_{\min }, \alpha_{\max }\right]$ in the following way:

$$
\begin{aligned}
& \operatorname{IF} \boldsymbol{S}^{(k-1)^{T}} D_{k}^{-1} z^{(k-1)} \leq 0 \text { THEN } \\
& \alpha_{k}^{(1)}=\min \left\{10 \cdot \alpha_{k-1}, \alpha_{\max }\right\} ; \\
& \text { ELSE } \\
& \alpha_{k}^{(1)}=\min \left\{\alpha_{\max }, \max \left\{\alpha_{\min }, \alpha_{k}^{(B B 1)}\right\}\right\} ; \\
& \text { ENDIF } \\
& \operatorname{IF} \boldsymbol{S}^{(k-1)^{T}} D_{k} z^{(k-1)} \leq 0 \text { THEN } \\
& \alpha_{k}^{(2)}=\min \left\{10 \cdot \alpha_{k-1}, \alpha_{\max }\right\} ; \\
& \text { ELSE } \\
& \alpha_{k}^{(2)}=\min \left\{\alpha_{\max }, \max \left\{\alpha_{\min }, \alpha_{k}^{(B B 2)}\right\}\right\} ; \\
& \text { ENDIF }
\end{aligned}
$$

the recent literature on steplength selection in gradient methods propose that steplength updating rules be designed by alternating the two BB formulae (Serafini et al. 2005; Zhou et al. 2006). In the case of nonscaled gradient methods (i.e., $D_{k}=I$ ) where the inequality $\alpha_{k}^{(B B 2)} \leq \alpha_{k}^{(B B 1)}$ holds (Serafini et al. 2005), remarkable convergence rate improvements have been obtained by alternation strategies that force the selection to be made in a suitable order of both low and high BB values. In Frassoldati et al. (2008), this aim is realized by an alternation criterion, which compares well with other popular BB-like steplength rules, namely

$$
\begin{aligned}
& \text { IF } \alpha_{k}^{(2)} / \alpha_{k}^{(1)} \leq \tau_{k} \text { THEN } \\
& \quad \alpha_{k}=\min _{j=\max \left\{1, k+1-M_{\alpha}\right\}, \ldots, k} \alpha_{j}^{(2)} ;
\end{aligned}
$$

$$
\begin{aligned}
& \tau_{k+1}=0.9 \cdot \tau_{k} ; \\
& \alpha_{k}=\alpha_{k}^{(1)} ; \quad \tau_{k+1}=1.1 \cdot \tau_{k} ;
\end{aligned}
$$

where $M_{\alpha}$ is a prefixed positive integer and $\tau_{1} \in(0,1)$. When scaled versions of the BB rules given in Eqs. (7)-(8) are used, the inequality $\alpha_{k}^{(B B 2)} \leq \alpha_{k}^{(B B 1)}$ is not always true. Nevertheless, a wide computational study suggests that this alternation criterion is more suitable in terms of convergence rate than the use of a single BB rule (Bonettini et al. 2009; Favati et al. 2010; Zanella et al. 2009). Furthermore, in our experience, the use of the BB values provided by Eq. (9) in the first iterations slightly improves the reconstruction accuracy and, consequently, in the proposed SGP version we start the steplength alternation only after the first 20 iterations.

When selecting the scaling matrix $D_{k}$, a suitable updating rule generally depends on the special form of the objective function. In our case, we chose the scaling matrix suggested by the RL algorithm, i.e.,

$D_{k}=\operatorname{diag}\left(\min \left[L_{2}, \max \left\{L_{1}, \boldsymbol{f}^{(k)}\right\}\right]\right)$,

where $L_{1}, L_{2}$ are prefixed thresholds.

\subsection{Single image deconvolution}

The problem of single image deconvolution in the presence of photon counting noise is the minimization of the KL divergence defined in Eq. (2) and the solution is given by the iterative RL algorithm of Eq. (1). When applying SGP, we only need the expression of the gradient of the KL divergence, which is given by (when the normalization of the PSF to unit volume is used)

$\nabla J_{0}(f ; g)=\mathbf{1}-A^{T} \frac{\boldsymbol{g}}{A \boldsymbol{f}+\boldsymbol{b}}$.

The SGP behavior with respect to RL was previously investigated in Bonettini et al. (2009).

\subsection{Multiple image deconvolution}

Successful multiple image deconvolution is fundamental to the future Fizeau interferometer of LBT called LINC-NIRVANA (Herbst et al. 2003) or to the "co-adding" method of images with different PSFs proposed by Lucy \& Hook (1992).

We define $p$ to be the number of detected images $\boldsymbol{g}_{j},(j=1$, $\ldots, p)$, with corresponding PSFs $\boldsymbol{K}_{j}$, all normalized to unit volume, and $A_{j} \boldsymbol{f}=\boldsymbol{K}_{j} * \boldsymbol{f}$. It is quite natural to assume that the $p$ images are statistically independent, such that the likelihood of the problem is the product of the likelihoods of the different images. If we assume again Poisson statistics, and we take the negative logarithm of the likelihood, then the maximization of the likelihood is equivalent to the minimization of a data-fidelity function, which is the sum of KL divergences, one for each image, i.e.

$$
\begin{array}{r}
J_{0}(\boldsymbol{f} ; \boldsymbol{g})=\sum_{j=1}^{p} \sum_{\mathbf{m} \in S}\left\{\boldsymbol{g}_{j}(\mathbf{m}) \ln \frac{\boldsymbol{g}_{j}(\mathbf{m})}{\left(A_{j} \boldsymbol{f}\right)(\mathbf{m})+\boldsymbol{b}_{j}(\mathbf{m})}\right. \\
\left.+\left(A_{j} \boldsymbol{f}\right)(\mathbf{m})+\boldsymbol{b}_{j}(\mathbf{m})-\boldsymbol{g}_{j}(\mathbf{m})\right\} .
\end{array}
$$


Algorithm 2 Ordered subset expectation maximization (OSEM)
method Choose the starting point $\boldsymbol{f}^{(0)}>0$.

FOR $k=0,1,2, \ldots$ DO THE FOLLOWING STEPS:

STEP 1. Set $\boldsymbol{h}^{(0)}=\boldsymbol{f}^{(k)}$;

SteP 2. For $j=1, \ldots, p$ compute

$$
\boldsymbol{h}^{(j)}=\boldsymbol{h}^{(j-1)} \circ\left(A_{j}^{\mathrm{T}} \frac{\boldsymbol{g}_{j}}{A_{j} \boldsymbol{h}^{(j-1)}+\boldsymbol{b}_{j}}\right) ;
$$

STEP 3. Set $\boldsymbol{f}^{(k+1)}=\boldsymbol{h}^{(p)}$.

$\underline{\text { END }}$

If we apply the standard expectation maximization method (Shepp \& Vardi 1982) to this problem, we obtain the iterative algorithm

$\boldsymbol{f}^{(k+1)}=\frac{1}{p} \boldsymbol{f}^{(k)} \circ \sum_{j=1}^{p} A_{j}^{\mathrm{T}} \frac{\boldsymbol{g}_{j}}{A_{j} \boldsymbol{f}^{(k)}+\boldsymbol{b}_{j}}$,

which we call the multiple image RL method (multiple RL, for short). Since the gradient of (12) is given by

$\nabla J_{0}(\boldsymbol{f} ; \boldsymbol{g})=\sum_{j=1}^{p}\left\{\mathbf{1}-A_{j}^{\mathrm{T}} \frac{\boldsymbol{g}_{j}}{A_{j} \boldsymbol{f}+\boldsymbol{b}_{j}}\right\}$,

we find that the algorithm presented in Eq. (13) is a scaled gradient method, with a scaling given, at iteration $k$, by $f^{(k)} / p$. Therefore, the application of SGP to this problem is straightforward.

However, for the reconstruction of LINC-NIRVANA images, we must consider that an acceleration of the algorithm in Eq. (13) is proposed in Bertero \& Boccacci (2000) by exploiting an analogy between the images of the interferometer and the projections in tomography. In this approach called OSEM (ordered subset expectation maximization, Hudson \& Larkin 1994), the sum over the $p$ images in Eq. (13) is replaced by a cycle over the same images. To avoid oscillations of the reconstructions within the cycle, a preliminary step is the normalization of the different images to the same flux, if different integration times are used in the acquisition process. The method OSEM is summarized in algorithm 2 .

As follows from practice and theoretical remarks, this approach reduces the number of iterations by a factor $p$. However, the computational cost of one multiple RL iteration is lower than that of one OSEM iteration: we need $3 p+1$ FFTs in the first case and $4 p$ FFTs in the second. In conclusion, the increase in efficiency provided by OSEM is roughly given by $(3 p+1) / 4$. When $p=3$ (the number of images provided by the interferometer will presumably be small), the efficiency is higher by a factor of 2.5 , and a factor of 4.7 when $p=6$. These results must be taken into account when considering the increase in the efficiency of SGP with respect to multiple RL. We can add that the convergence of SGP is proven while that of OSEM is not, even if it has always been verified in our numerical experiments.

\subsection{Boundary effect correction}

If the target $f$ is not completely contained in the image domain, then the previous deconvolution methods produce annoying boundary artifacts. It is not the purpose of this paper to discuss the different methods for solving this problem. We focus on an approach proposed in Bertero \& Boccacci (2005) for single image deconvolution and in Anconelli et al. (2006) for multiple image deconvolution. Here we present the equations in the case of multiple images, where a single image corresponds to $p=1$.

The idea is to reconstruct the object $f$ over a domain broader than that of the detected images and to merge, by zero padding, the arrays of the images and the object into arrays of dimensions that enable their Fourier transform to be computed by means of FFT. We denote by $\bar{S}$ the set of values of the multi-index labeling the pixels of the broader arrays containing $S$ and by $R$ that of the object array contributing to $S$, such that $S \subset R \subset \bar{S}$. It is also obvious that also the PSFs must be defined over $\bar{S}$ and that this can be done in different ways, depending on the specific problem one is considering. We point out that they must be normalized to unit volume over $\bar{S}$. We also note that $R$ corresponds to the part of the object contributing to the detected images and that it depends on the extent of the PSFs. It can be estimated from this information as we indicate in the following (see Eq. (20)). The reconstruction of $\boldsymbol{f}$ outside $S$, is unreliable in most cases, but its reconstruction inside $S$ is practically free of boundary artifacts, as shown in the papers cited above and in the experiments of Sect. 4.

If we denote by $\boldsymbol{M}_{R}, \boldsymbol{M}_{S}$ the arrays, defined over $\bar{S}$, which are 1 over $R, S$ respectively and 0 outside, we define the matrices $A_{j}$ and $A_{j}^{\mathrm{T}}$

$\left(A_{j} \boldsymbol{f}\right)(\mathbf{m})=\boldsymbol{M}_{S}(\mathbf{m}) \sum_{\mathbf{n} \in \bar{S}} \boldsymbol{K}_{j}(\mathbf{m}-\mathbf{n}) \boldsymbol{M}_{R}(\mathbf{n}) \boldsymbol{f}(\mathbf{n})$,
$\left(A_{j}^{\mathrm{T}} \boldsymbol{g}\right)(\mathbf{n})=\boldsymbol{M}_{R}(\mathbf{n}) \sum_{\mathbf{m} \in \bar{S}} \boldsymbol{K}_{j}(\mathbf{m}-\mathbf{n}) \boldsymbol{M}_{S}(\mathbf{m}) \boldsymbol{g}(\mathbf{m})$.

In the second equation, $\boldsymbol{g}$ denotes a generic array defined over $\bar{S}$. Both matrices can be easily computed by means of FFT. With these definitions, the data fidelity function is then given again by Eq. (12), with $S$ replaced by $\bar{S}$, while its gradient is now given by

$\nabla J_{0}(\boldsymbol{f} ; \boldsymbol{g})=\sum_{j=1}^{p}\left\{A_{j}^{\mathrm{T}} \mathbf{1}-A_{j}^{\mathrm{T}} \frac{\boldsymbol{g}_{j}}{A_{j} \boldsymbol{f}+\boldsymbol{b}_{j}}\right\}$,

leading to the introduction of the functions

$\boldsymbol{\alpha}_{j}(\mathbf{n})=\sum_{\mathbf{m} \in \bar{S}} \boldsymbol{K}_{j}(\mathbf{m}-\mathbf{n}) \boldsymbol{M}_{S}(\mathbf{m}) \boldsymbol{g}(\mathbf{m})$,

$\boldsymbol{\alpha}(\mathbf{n})=\sum_{j=1}^{p} \boldsymbol{\alpha}_{j}(\mathbf{n}), \quad \boldsymbol{n} \in \bar{S}$.

These functions can be used to define the reconstruction domain $R$, since they can be either very small or zero in pixels of $\bar{S}$, depending on the behavior of the PSFs. Given a thresholding value $\sigma$, we use the definition

$R=\left\{\boldsymbol{n} \in \bar{S} \mid \boldsymbol{\alpha}_{j}(\boldsymbol{n}) \geq \sigma ; j=1, \ldots, p\right\}$.

The RL algorithm, with boundary effect correction, is then given by

$\boldsymbol{f}^{(k+1)}=\frac{\boldsymbol{M}_{R}}{\boldsymbol{\alpha}} \circ \boldsymbol{f}^{(k)} \circ \sum_{j=1}^{p} A_{j}^{\mathrm{T}} \frac{\boldsymbol{g}_{j}}{A_{j} \boldsymbol{f}^{(k)}+\boldsymbol{b}_{j}}$,

the quotient being zero in the pixels outside $R$. Similarly, the OSEM algorithm, with a boundary effect correction is given by algorithm 2 where Eq. (15) is replaced by

$\boldsymbol{h}^{(j)}=\frac{\boldsymbol{M}_{R}}{\boldsymbol{\alpha}_{j}} \circ \boldsymbol{h}^{(j-1)} \circ\left(A_{j}^{\mathrm{T}} \frac{\boldsymbol{g}_{j}}{A_{j} \boldsymbol{h}^{(j-1)}+\boldsymbol{b}_{j}}\right)$. 
As far as the SGP algorithm concerns, the boundary effect correction is incorporated to the scaling matrix

$D_{k}=\operatorname{diag}\left(\frac{\boldsymbol{M}_{R}}{\boldsymbol{\alpha}} \circ \min \left[L_{2}, \max \left\{L_{1}, \boldsymbol{f}^{(k)}\right\}\right]\right)$

while all the other steps remain unchanged.

\section{Computational features}

The description of the SGP algorithm provided in Sect. 2.1 indicates several ingredients on which the success of the recipe depends: the choice of the starting point, the selection of the parameters defining the method, and the stopping criterion. In the following, we briefly describe which choices were made in our numerical experimentation, and comment on the parallel implementation of the algorithm.

\subsection{Initialization}

As far as the SGP initial point $\boldsymbol{f}^{(0)}$ concerns, any non-negative image is allowed. The possible choices implemented in our code are:

- the null image $\boldsymbol{f}^{(0)}=\mathbf{0}$;

- the noisy image $\boldsymbol{g}$ (or, in the case of multiple deconvolution, the noisy image $\boldsymbol{g}_{1}$ corresponding to the first PSF $\boldsymbol{K}_{1}$ );

- a constant image with pixel values equal to the backgroundsubtracted flux (or mean flux in the case of multiple deconvolution) of the noisy data divided by the number of pixels. If the boundary effect correction is considered, only the pixels in the object array $R$ become equal to this constant, while the remaining values of $\bar{S}$ are set to zero. In future extensions of our codes, the constant image will be convolved with a Gaussian to avoid the presence of sharp edges;

- any input image provided by the user.

The constant image $f^{(0)}$ was chosen for our numerical experiments, which is also the initial point used for RL.

\subsection{SGP parameter setting}

Even if the number of SGP parameters is certainly higher than those of the RL and OSEM approaches, the huge amount of tests carried out in several applications has led to an optimization of these values, which allows the user to have at his disposal a robust approach without the need for any problem-dependent parameter tuning. In our present study, some of these values were fixed according to the original paper of Bonettini et al. (2009), as in the case of the line-search parameters $\beta$ and $\theta$, which were set to $10^{-4}$ and 0.4 , respectively. In addition, most of the steplength parameters remained unchanged, as $\alpha_{0}=1.3$, $\tau_{1}=0.5, \alpha_{\max }=10^{5}$, and $M_{\alpha}=3$, while $\alpha_{\text {min }}$ was set to $10^{-5}$.

The main change concerned the choice of the bounds $\left(L_{1}, L_{2}\right)$ for the scaling matrices. While in the original paper, the choice was a couple of fixed values $\left(10^{-10}, 10^{10}\right)$, independent of the data, we decided to automatically adapt these bounds to the input image: we performed one step of the RL method and tuned the parameters $\left(L_{1}, L_{2}\right)$ according to the $\min / \max$ positive values $y_{\min } / y_{\max }$ of the resulting image according to the rule

IF $y_{\max } / y_{\min }<50$ THEN

$L_{1}=y_{\min } / 10$

$L_{2}=y_{\max } \cdot 10$

ELSE

$$
\begin{aligned}
& L_{1}=y_{\min } ; \\
& L_{2}=y_{\max } ;
\end{aligned}
$$$$
\text { ENDIF }
$$

\subsection{Stopping rules}

As mentioned in the introduction, in many instances both RL and SGP must not be pushed to convergence and an early stopping of the iterations is required to achieve reasonable reconstructions. In our code, we introduced different stopping criteria, which can be adapted by the user according to his/her purposes:

- fixed number of iterations. The user can decide how many iterations of SGP must be done;

- convergence of the algorithm. In such a case, a stopping criterion based on the convergence of the data-fidelity function to its minimum value is introduced. Iteration is stopped when

$\left|J_{0}\left(\boldsymbol{f}^{(k+1)} ; \boldsymbol{g}\right)-J_{0}\left(\boldsymbol{f}^{(k)} ; \boldsymbol{g}\right)\right| \leq$ tol $J_{0}\left(\boldsymbol{f}^{(k)} ; \boldsymbol{g}\right)$,

where tol is a parameter that can be selected by the user;

- minimization of the reconstruction error. This criterion can be used in a simulation study. If one knows the object $\widetilde{f}$ used to generate the synthetic images, then one can stop the iterations when the relative reconstruction error

$\rho^{(k)}=\frac{\left|\boldsymbol{f}^{(k)}-\widetilde{\boldsymbol{f}}\right|}{|\widetilde{\boldsymbol{f}}|}$

reaches a minimum value. A very frequently used measure of error is given by the $\ell_{2}$ norm, i.e. $|\cdot|=\|\cdot\|_{2}$ and this is the criterion implemented in our code;

- the use of a discrepancy criterion. In the case of real data, one can use a given value of some measure of the "distance" between the real data and the data computed by means of the current iteration. A recently proposed criterion consists in defining the "discrepancy function" for $p$ images $\boldsymbol{g}_{j}$ of size $N \times N$

$\mathcal{D}^{(k)}=\frac{2}{p N^{2}} J_{0}\left(\boldsymbol{f}^{(k)} ; \boldsymbol{g}\right)$,

and stopping the iterations when $\mathcal{D}^{(k)}=b$, where $b$ is a given number close to 1 . Work is in progress to validate this discrepancy criterion with the purpose of obtaining rules of thumb for estimating $b$ in real applications.

The last stopping rule deserves a few comments. In Bertero et al. (2010), it is shown that, for a single image, if $\widetilde{f}$ is the object generating the noisy image $\boldsymbol{g}$, then the expected value of $J_{0}(\widetilde{f} ; \boldsymbol{g})$ is close to $N^{2} / 2$. This property is used to select a value of the regularization parameter when the image reconstruction problem is formulated as the minimization of the KL divergence with the addition of a suitable regularization term. This use is justified by evidence that in some important cases it provides a unique value of the regularization parameter. Moreover, it has also been shown that the quantity $\mathcal{D}^{(k)}$, defined in Eq. (26), decreases for increasing $k$, starting from a value greater than 1 . Therefore, it can be used as a stopping criterion. Preliminary numerical experiments described in that paper show that it can provide a sensible stopping rule at least in simulation studies.

\subsection{IDL and GPU implementation}

Our implementation of the SGP algorithm was written in IDL, a well-known and frequently used language in astronomical environments. This data-analysis programming language is wellsuited to work with images, using optimized built-in vector operations. Nevertheless, it is not intended that usability should be compromised by performance. 
As already shown in Ruggiero et al (2010), the C++ implementation of the SGP algorithm is well-suited to parallelization and good computational speedup is obtained exploiting the CUDA technology. The CUDA is a framework developed by NVIDIA that enables the use of GPU for programming. These graphics cards are nowadays in many personal computers and their core is highly parallel, consisting of several hundreds of computational units. Many recent applications show that the increase in efficiency achieved with this technology is significant and its cost is much lower than that of a medium-sized cluster. We note that memory management is crucial to ensure the optimal performance when using GPU. The transfer speed of data from central memory to GPU is much slower than the GPU-toGPU transfer, hence to maximize the GPU benefits it is very important to reduce the CPU-to-GPU memory communications and retain all the problem data in the GPU memory.

The CUDA technology is available in IDL as part of GPUlib, a software library that enables GPU-accelerated calculations, developed by Tech-X Corporation. It has to be noted that the FFT routine included in the current version of GPUlib (1.4.4) is available only in single precision. Results from this function differ slightly from the ones obtained in double precision by IDL, causing some numerical differences in our experiments.

\section{Results}

We now demonstrate, by means of a few numerical experiments, the effectiveness of the SGP algorithm and its IDL-based GPU implementation in the solution of the deblurring problems described in Sect. 2. Our test platform consists in a personal computer equipped with an AMD Athlon X2 Dual-Core at $3.11 \mathrm{GHz}, 3 \mathrm{~GB}$ of RAM, and the graphics processing unit NVIDIA GTX 280 with CUDA 3.2. We consider CPU implementations of RL, SGP, and OSEM in IDL 7.0; the GPU implementations are developed in mixed IDL and CUDA language by means of the GPUlib 1.4.4.

The set of numerical experiments can be divided into two groups: single image and multiple image deconvolution. For each group, some tests on boundary effect correction are included.

\subsection{Single image deconvolution}

The first experiments are based on $256 \times 256$ HST images of the planetary nebula NGC 7027, the galaxy NGC 6946 and the Crab nebula NGC 19521. We use three different integrated magnitudes $(m)$ of 10,12 , and 15 , not corresponding to the effective magnitudes of these objects but introduced for obtaining simulated images with different noise levels. In Fig. 1 we show the three objects in the left panels. In the following, they are denoted nebula, galaxy and Crab.

These objects are convolved with an AO-corrected PSF $^{1}$ shown in Fig. 2 without zoom, and frequently used in numerical experiments. The parameters of this PSF (pixel size, diameter of the telescope, etc.) are not provided. However, it has approximately the same width as the ideal PSF used in the third experiment reported below and simulated assuming a telescope of $8.25 \mathrm{~m}$, a wavelength of $2.2 \mu \mathrm{m}$, and a pixel size of 5 mas.

A background of about $13.5 \mathrm{mag} \operatorname{arcsec}^{-2}$, corresponding to observations in $K$-band, is added to the blurred images and the results are perturbed with Poisson noise and additive Gaussian

\footnotetext{
1 Downloaded from http://www.mathcs.emory.edu/ nagy/ RestoreTools/index.html
}

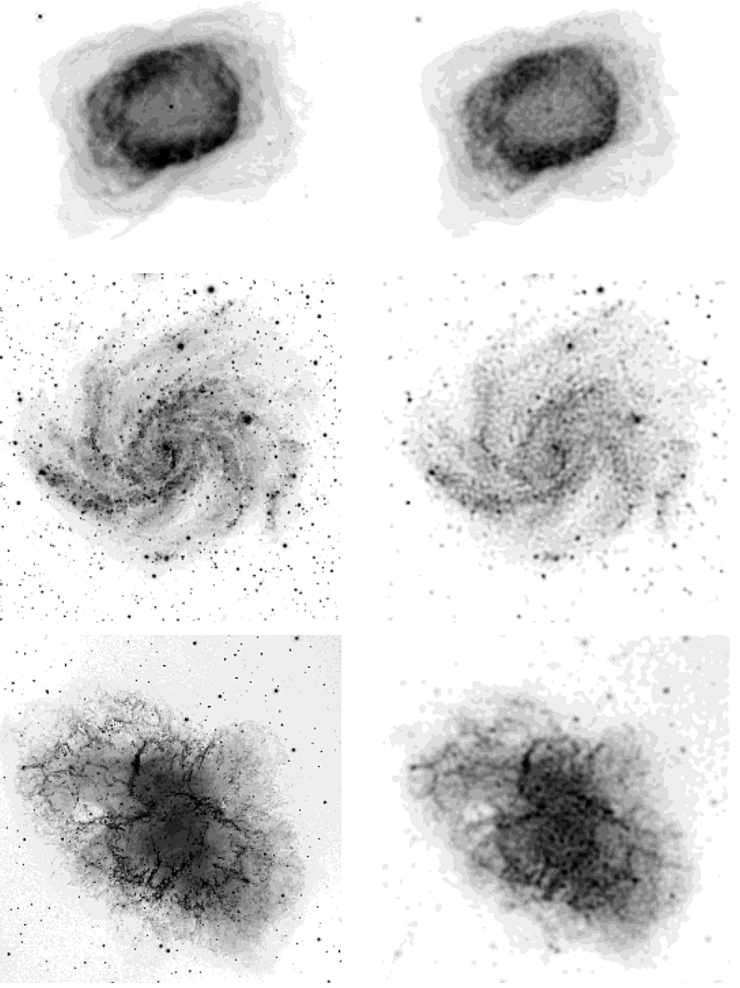

Fig. 1. The three objects, represented with reverse gray scale (left panels; from up to down nebula, galaxy and $\mathrm{Crab}$ ), and the reconstructions with minimum relative rms error ( $m=15$; right panels).

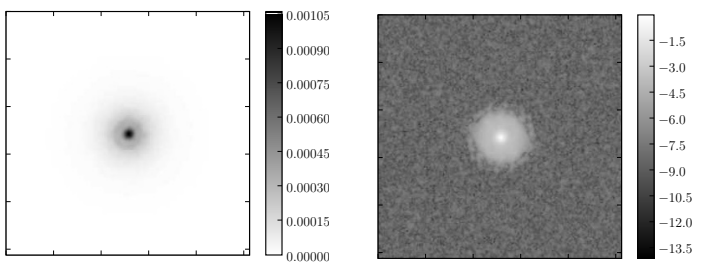

Fig. 2. The PSF used in the experiments of single image deconvolution (left panel), represented with reverse gray scale, and the corresponding MTF (right panel).

noise with $\sigma=10 \mathrm{e}^{-} / \mathrm{px}$. According to the approach proposed in Snyder et al. (1994), compensation for readout noise is obtained in the deconvolution algorithms by adding the constant $\sigma^{2}=100$ to the images and the background. In Table 1, the performances of RL and SGP are reported, in terms of iteration numbers needed to obtain the minimum relative rms error, CPU times, and speedups provided by the two GPU versions with respect to the serial ones. The reconstructions corresponding to the minimum relative rms error, in the case $m=15$, are shown in the right panels of Fig. 1.

In the second experiment, we use the same datasets created in the previous one to test the effectiveness of the procedure described in Sect. 2.4 for the reduction of boundary effects. To this aim, the $256 \times 256$ blurred and noisy images are partitioned into four partially overlapping $160 \times 160$ subdomains. Each one of the four partial images is merged, by zero-padding, in a $256 \times 256$ array that is used, together with the original $256 \times$ 256 PSF, for the reconstruction of the four parts of the object by means of the RL and SGP algorithms with boundary effect 
M. Prato et al.: Efficient deconvolution methods for astronomical imaging: Algorithms and IDL-GPU codes

Table 1. Iteration numbers, relative rms errors, computational times, and speedups of RL and SGP, provided by the corresponding GPU implementations, for the three $256 \times 256$ objects nebula, galaxy and Crab.

\begin{tabular}{|c|c|c|c|c|c|c|c|c|c|c|c|c|}
\hline \multirow[b]{2}{*}{ Algorithm } & \multicolumn{4}{|c|}{ Nebula $(m=10)$} & \multicolumn{4}{|c|}{ Galaxy $(m=10)$} & \multicolumn{4}{|c|}{ Crab $(m=10)$} \\
\hline & It & Err & $\mathrm{Sec}$ & SpUp & It & Err & $\mathrm{Sec}$ & SpUp & It & Err & $\mathrm{Sec}$ & SpUp \\
\hline RL & 528 & 0.021 & 41.28 & - & $10000^{*}$ & 0.140 & 795.3 & - & 5353 & 0.128 & 419.8 & - \\
\hline RL_CUDA & 528 & 0.021 & 2.079 & 19.9 & $10000^{*}$ & 0.140 & 35.09 & 22.7 & 5353 & 0.128 & 19.45 & 21.6 \\
\hline SGP & 50 & 0.021 & 4.719 & - & 406 & 0.141 & 38.61 & - & 151 & 0.129 & 14.28 & - \\
\hline \multirow[t]{2}{*}{ SGP_CUDA } & 50 & 0.021 & 0.344 & 13.7 & 406 & 0.142 & 3.313 & 11.7 & 151 & 0.129 & 1.219 & 11.7 \\
\hline & \multicolumn{4}{|c|}{ Nebula $(m=12)$} & \multicolumn{4}{|c|}{ Galaxy $(m=12)$} & \multicolumn{4}{|c|}{$\operatorname{Crab}(m=12)$} \\
\hline Algorithm & It & Err & $\mathrm{Sec}$ & SpUp & It & Err & $\mathrm{Sec}$ & SpUp & It & Err & $\mathrm{Sec}$ & SpUp \\
\hline RL & 124 & 0.026 & 9.797 & - & 3887 & 0.157 & 304.6 & - & 954 & 0.136 & 74.83 & - \\
\hline RL_CUDA & 124 & 0.026 & 0.516 & 19.0 & 3887 & 0.157 & 14.50 & 21.0 & 954 & 0.136 & 3.516 & 21.3 \\
\hline SGP & 24 & 0.026 & 2.344 & - & 153 & 0.159 & 14.42 & - & 52 & 0.137 & 4.984 & - \\
\hline \multirow[t]{2}{*}{ SGP_CUDA } & 24 & 0.026 & 0.203 & 11.5 & 153 & 0.159 & 1.266 & 11.4 & 52 & 0.137 & 0.406 & 12.3 \\
\hline & \multicolumn{4}{|c|}{ Nebula $(m=15)$} & \multicolumn{4}{|c|}{ Galaxy $(m=15)$} & \multicolumn{4}{|c|}{ Crab $(m=15)$} \\
\hline Algorithm & It & Err & $\mathrm{Sec}$ & SpUp & It & Err & $\mathrm{Sec}$ & SpUp & It & Err & $\mathrm{Sec}$ & SpUp \\
\hline RL & 124 & 0.063 & 9.766 & - & 448 & 0.234 & 35.14 & - & 128 & 0.172 & 10.09 & - \\
\hline RL_CUDA & 124 & 0.063 & 0.469 & 20.8 & 448 & 0.234 & 1.594 & 22.0 & 128 & 0.172 & 0.483 & 20.9 \\
\hline SGP & 12 & 0.060 & 1.250 & - & 21 & 0.234 & 2.094 & - & 10 & 0.172 & 1.093 & - \\
\hline SGP_CUDA & 12 & 0.060 & 0.109 & 11.5 & 21 & 0.234 & 0.156 & 13.4 & 10 & 0.172 & 0.093 & 11.8 \\
\hline
\end{tabular}

Notes. Iterations are stopped at a minimum relative rms error in the serial algorithms (the asterisks denote the maximum number of iterations allowed).

Table 2. Reconstruction of nebula, galaxy, and Crab as a mosaic of the reconstructions of four subimages with boundary effect correction.

\begin{tabular}{|c|c|c|c|c|c|c|c|c|c|c|c|c|}
\hline \multirow[b]{2}{*}{ Algorithm } & \multicolumn{4}{|c|}{ Nebula $(m=10)$} & \multicolumn{4}{|c|}{ Galaxy $(m=10)$} & \multicolumn{4}{|c|}{ Crab $(m=10)$} \\
\hline & It & Err & $\mathrm{Sec}$ & SpUp & It & Err & $\mathrm{Sec}$ & SpUp & It & Err & $\mathrm{Sec}$ & SpUp \\
\hline RL & 818 & 0.021 & 243.8 & - & $10000^{*}$ & 0.144 & 2813 & - & 4070 & 0.129 & 1146 & - \\
\hline RL_CUDA & 818 & 0.021 & 12.16 & 20.0 & $10000^{*}$ & 0.144 & 141.5 & 19.9 & 4070 & 0.129 & 61.55 & 18.6 \\
\hline SGP & 96 & 0.022 & 35.16 & - & 435 & 0.144 & 171.6 & - & 129 & 0.129 & 46.42 & - \\
\hline \multirow[t]{2}{*}{ SGP_CUDA } & 96 & 0.022 & 3.406 & 10.3 & 435 & 0.148 & 14.41 & 11.9 & 129 & 0.133 & 4.342 & 10.7 \\
\hline & \multicolumn{4}{|c|}{ Nebula $(m=12)$} & \multicolumn{4}{|c|}{ Galaxy $(m=12)$} & \multicolumn{4}{|c|}{ Crab $(m=12)$} \\
\hline Algorithm & It & Err & $\mathrm{Sec}$ & SpUp & It & Err & $\mathrm{Sec}$ & SpUp & It & Err & $\mathrm{Sec}$ & SpUp \\
\hline RL & 127 & 0.026 & 38.42 & - & 2347 & 0.160 & 696.9 & - & 696 & 0.137 & 196.5 & - \\
\hline RL_CUDA & 127 & 0.026 & 2.108 & 18.2 & 2347 & 0.160 & 35.13 & 19.8 & 696 & 0.137 & 10.99 & 17.9 \\
\hline SGP & 21 & 0.026 & 9.563 & - & 126 & 0.161 & 51.11 & - & 53 & 0.137 & 19.41 & - \\
\hline \multirow[t]{2}{*}{ SGP_CUDA } & 21 & 0.026 & 0.874 & 10.9 & 126 & 0.161 & 4.438 & 11.5 & 53 & 0.137 & 1.922 & 10.1 \\
\hline & \multicolumn{4}{|c|}{ Nebula $(m=15)$} & \multicolumn{4}{|c|}{ Galaxy $(m=15)$} & \multicolumn{4}{|c|}{$\operatorname{Crab}(m=15)$} \\
\hline Algorithm & It & Err & $\mathrm{Sec}$ & SpUp & It & Err & $\mathrm{Sec}$ & SpUp & It & Err & $\mathrm{Sec}$ & SpUp \\
\hline RL & 96 & 0.064 & 27.58 & - & 297 & 0.234 & 89.22 & - & 99 & 0.172 & 28.08 & - \\
\hline RL_CUDA & 96 & 0.064 & 1.703 & 16.2 & 297 & 0.234 & 4.547 & 19.6 & 99 & 0.172 & 1.704 & 16.5 \\
\hline SGP & 10 & 0.061 & 4.234 & - & 17 & 0.236 & 7.375 & - & 9 & 0.172 & 3.859 & - \\
\hline SGP_CUDA & 10 & 0.061 & 0.407 & 10.4 & 17 & 0.236 & 0.657 & 11.2 & 9 & 0.172 & 0.360 & 10.7 \\
\hline
\end{tabular}

Notes. The number of iterations is the one required for reconstructing each subdomain, while the reported computational time is the total time required for the 4 reconstructions.

correction. From the four reconstructions $128 \times 128$, nonoverlapping images are extracted and the complete reconstructed image is formed as a mosaic of them. An example of the result is shown in Fig. 3. By comparing with the reconstruction of the full image, it is clear that the mosaic of the four reconstructions does not exhibit visible boundary effects.

The results of this experiment for the three objects are reported in Table 2. The reconstruction error is the relative rms error between the mosaic and the original object. By comparing with the results of Table 1, we find that the procedure does not significantly increase the reconstruction error. We also point out that we choose the number of iterations corresponding to the global minimum, i.e. that providing the best performance on the mosaic of the four reconstructions obtained in the four subdomains. The computational time is the total time of the four reconstructions.

The third experiment intends to investigate the speedups achievable by SGP when varying the size of the images. We adopt the same procedure used in Ruggiero et al. (2010). The original $256 \times 256$ objects are convolved with an ideal PSF (described in the second paragraph of Sect. 4.1) and perturbed with background and Poisson noise. Next, images with a larger size are obtained by a Fourier-based re-binning, i.e. the FFT of the original image is expanded by zero padding to a double-sized array and the zero frequency component is multiplied by four. In this way, the background and the noise level are approximately unchanged and no new content is introduced at high frequencies. In particular, no out-of-band noise is introduced and therefore the number of iterations needed to converge to the best solution is probably underestimated, since we use, for any size, the number derived in the case $256 \times 256$. In this experiment, we consider only the nebula and the galaxy with two magnitudes 10 and 15 . The original images are expanded up to a size of $2048 \times 2048$. The results are reported in Tables 3 and 4, where we highlighted both the speedup observed between GPU and serial implementations (labeled "Par") and the one provided by the 

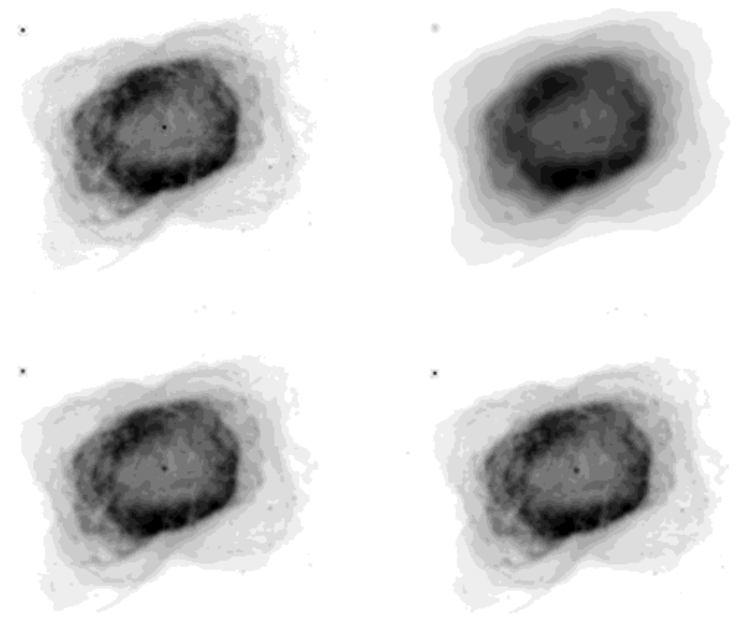

Fig. 3. Upper-left panel: the original nebula; upper-right panel: its blurred and noisy image in the case $m=10$; lower left panel: reconstruction of the global image; lower-left panel: reconstruction as a mosaic of four reconstructions of partially overlapping subdomains, using the algorithms with boundary effect correction.

use of SGP instead of RL (labeled "Alg"). We note that the computational gain achieved by the parallel architecture increases in proportion to the size of the image. As far as the speedup of $\mathrm{SGP}$ with respect to RL is concerned, strong problem-dependent differences in the number of iterations required to reach the minimum errors do not lead to a similarly regular behavior.

\subsection{Multiple images}

We test the efficiency of three algorithms for multiple image deconvolution, i.e. multiple RL, OSEM, and SGP (applied to multiple RL), by means of simulated images of the Fizeau interferometer LINC-NIRVANA (LN, for short; Herbst et al. 2003) of the LBT. The LN is in an advanced realization phase by a consortium of German and Italian institutions, led by the Max Planck Institute for Astronomy in Heidelberg. It is a true imager with a maximum baseline of $22.8 \mathrm{~m}$, thus producing images with anisotropic resolution: that of a $22.8 \mathrm{~m}$ telescope in the direction of the baseline and that of a $8.4 \mathrm{~m}$ (the diameter of LBT mirrors) in the orthogonal direction. By acquiring images with different orientations of the baseline and applying suitable deconvolution methods, it is possible, in principle, to achieve the resolution of a $22.8 \mathrm{~m}$ telescope in all directions. The LN will be equipped with a detector consisting of $2048 \times 2048$ pixels with a pixel size of about 5 mas, corresponding to a F0V of $10^{\prime \prime} \times 10^{\prime \prime}$ for each orientation of the baseline. Since in $K$-band the resolution of a $22.8 \mathrm{~m}$ mirror is about 20 mas, the detector provides an oversampling of a factor four.

In our simulations, we use PSFs generated with the code LOST (Arcidiacono et al. 2004); one of them, with SR $=70 \%$ and horizontal baseline, is shown in Fig. 4 together with the corresponding MTF. Moreover, we consider two test objects: one is again the nebula NGC 7027, with two magnitudes, 10 and 15, and size $512 \times 512$ (therefore, the images are noisier than those of the $256 \times 256$ version with the same integrated magnitude); the other is a model of an open star cluster based on an image of the Pleiades (star cluster, for short), consisting of 9 stars with magnitudes ranging from 12.86 to 15.64 . These objects are convolved with three PSFs corresponding to three equispaced
Table 3. Reconstruction of the nebula NGC 7027 with different image sizes.

\begin{tabular}{|c|c|c|c|c|c|}
\hline \multicolumn{6}{|c|}{$\bar{m}=10$} \\
\hline Algorithm & Size & Err & $\mathrm{Sec}$ & $\begin{array}{l}\text { SpUp } \\
\text { (Par) }\end{array}$ & $\begin{array}{l}\text { SpUp } \\
\text { (Alg) }\end{array}$ \\
\hline \multirow{4}{*}{$\begin{array}{l}\text { RL } \\
\text { It }=10000^{*}\end{array}$} & $256^{2}$ & 0.051 & 783.9 & - & - \\
\hline & $512^{2}$ & 0.051 & 4527 & - & - \\
\hline & $1024^{2}$ & 0.051 & 17610 & - & - \\
\hline & $2048^{2}$ & 0.051 & 80026 & - & - \\
\hline \multirow{4}{*}{$\begin{array}{l}\text { RL_CUDA } \\
\text { It }=10000^{*}\end{array}$} & $256^{2}$ & 0.051 & 35.63 & 22.0 & - \\
\hline & $512^{2}$ & 0.051 & 69.77 & 64.9 & - \\
\hline & $1024^{2}$ & 0.051 & 149.5 & 118 & - \\
\hline & $2048^{2}$ & 0.051 & 469.1 & 171 & - \\
\hline \multirow{4}{*}{$\begin{array}{l}\text { SGP } \\
\text { It }=272\end{array}$} & $256^{2}$ & 0.052 & 26.14 & - & 30.0 \\
\hline & $512^{2}$ & 0.051 & 143.6 & - & 31.5 \\
\hline & $1024^{2}$ & 0.051 & 554.0 & - & 31.8 \\
\hline & $2048^{2}$ & 0.051 & 2493 & - & 32.1 \\
\hline \multirow{4}{*}{$\begin{array}{l}\text { SGP_CUDA } \\
\text { It }=272\end{array}$} & $256^{2}$ & 0.052 & 1.797 & 14.5 & 19.8 \\
\hline & $512^{2}$ & 0.052 & 3.469 & 41.4 & 20.1 \\
\hline & $1024^{2}$ & 0.052 & 8.016 & 69.1 & 18.7 \\
\hline & $2048^{2}$ & 0.052 & 25.66 & 97.2 & 18.3 \\
\hline \multirow[b]{2}{*}{ Algorithm } & & $m=1$ & & & \\
\hline & Size & Err & $\mathrm{Sec}$ & $\begin{array}{c}\text { SpUp } \\
\text { (Par) }\end{array}$ & $\begin{array}{l}\text { SpUp } \\
\text { (Alg) }\end{array}$ \\
\hline \multirow{4}{*}{$\begin{array}{l}\mathrm{RL} \\
\mathrm{It}=612\end{array}$} & $256^{2}$ & 0.068 & 48.27 & - & - \\
\hline & $512^{2}$ & 0.064 & 278.7 & - & - \\
\hline & $1024^{2}$ & 0.062 & 1068 & - & - \\
\hline & $2048^{2}$ & 0.062 & 4897 & - & - \\
\hline \multirow{4}{*}{$\begin{array}{l}\text { RL_CUDA } \\
\text { It }=612\end{array}$} & $256^{2}$ & 0.068 & 2.219 & 21.8 & - \\
\hline & $512^{2}$ & 0.064 & 4.109 & 67.8 & - \\
\hline & $1024^{2}$ & 0.062 & 9.250 & 115 & - \\
\hline & $2048^{2}$ & 0.062 & 29.13 & 168 & - \\
\hline \multirow{4}{*}{$\begin{array}{l}\text { SGP } \\
\text { It }=31\end{array}$} & $256^{2}$ & 0.068 & 3.016 & - & 16.0 \\
\hline & $512^{2}$ & 0.064 & 16.95 & - & 16.4 \\
\hline & $1024^{2}$ & 0.062 & 65.22 & - & 16.4 \\
\hline & $2048^{2}$ & 0.061 & 290.8 & - & 16.8 \\
\hline \multirow{4}{*}{$\begin{array}{l}\text { SGP_CUDA } \\
\text { It }=31\end{array}$} & $256^{2}$ & 0.068 & 0.218 & 13.8 & 10.2 \\
\hline & $512^{2}$ & 0.064 & 0.421 & 40.3 & 9.76 \\
\hline & $1024^{2}$ & 0.062 & 1.063 & 61.4 & 8.70 \\
\hline & $2048^{2}$ & 0.061 & 3.406 & 85.4 & 8.55 \\
\hline
\end{tabular}

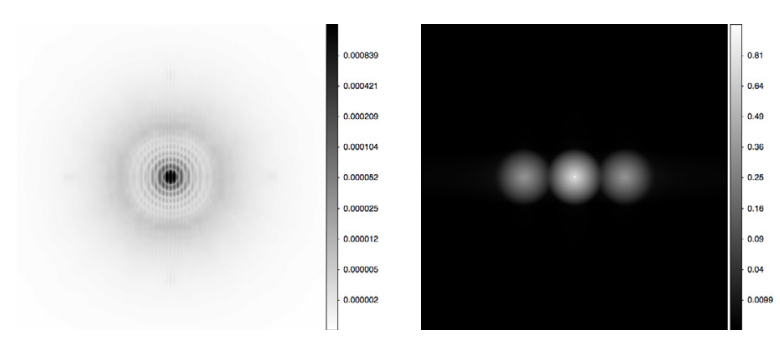

Fig. 4. Simulated PSF of LINC-NIRVANA with SR $=70 \%$ (left panel) and the corresponding MTF (right panel). The PSF is monochromatic in $K$-band and is the PSF of a $8.4 \mathrm{~m}$ mirror (the diameter of the two mirrors of LBT) modulated by the interferometric fringes. Accordingly, in the MTF the central disk corresponds to the band of a $8.4 \mathrm{~m}$ mirror while the two side disks are replicas due to interferometry.

orientations of the baseline, $0^{\circ}, 60^{\circ}$, and $120^{\circ}$. In the $u, v$ plane they provide a satisfactory coverage of the band of a $22.8 \mathrm{~m}$ telescope (see, for instance, Bertero et al. 2011, a review paper where the generation of the images used in this paper is described in greater detail). The results are perturbed with a 
M. Prato et al.: Efficient deconvolution methods for astronomical imaging: Algorithms and IDL-GPU codes

Table 4. Reconstruction of the galaxy NGC 6946 with different image sizes.

\begin{tabular}{|c|c|c|c|c|c|}
\hline \multicolumn{6}{|c|}{$\bar{~} m=10$} \\
\hline Algorithm & Size & Err & $\mathrm{Sec}$ & $\begin{array}{c}\text { SpUp } \\
\text { (Par) }\end{array}$ & $\begin{array}{l}\text { SpUp } \\
\text { (Alg) }\end{array}$ \\
\hline \multirow{4}{*}{$\begin{array}{l}\text { RL } \\
\text { It }=10000^{*}\end{array}$} & $256^{2}$ & 0.293 & 786.0 & - & - \\
\hline & $512^{2}$ & 0.293 & 4545 & - & - \\
\hline & $1024^{2}$ & 0.293 & 17402 & - & - \\
\hline & $2048^{2}$ & 0.293 & 80022 & - & - \\
\hline \multirow{4}{*}{$\begin{array}{l}\text { RL_CUDA } \\
\text { It }=10000^{*}\end{array}$} & $256^{2}$ & 0.293 & 36.64 & 21.5 & - \\
\hline & $512^{2}$ & 0.293 & 67.94 & 66.9 & - \\
\hline & $1024^{2}$ & 0.293 & 146.7 & 119 & - \\
\hline & $2048^{2}$ & 0.293 & 463.9 & 172 & - \\
\hline \multirow{4}{*}{$\begin{array}{l}\text { SGP } \\
\text { It }=928\end{array}$} & $256^{2}$ & 0.292 & 88.72 & - & 8.86 \\
\hline & $512^{2}$ & 0.291 & 484.3 & - & 9.38 \\
\hline & $1024^{2}$ & 0.291 & 1854 & - & 9.19 \\
\hline & $2048^{2}$ & 0.291 & 8386 & - & 9.54 \\
\hline \multirow{4}{*}{$\begin{array}{l}\text { SGP_CUDA } \\
\text { It }=928\end{array}$} & $256^{2}$ & 0.293 & 7.219 & 12.3 & 5.08 \\
\hline & $512^{2}$ & 0.293 & 11.14 & 43.5 & 6.10 \\
\hline & $1024^{2}$ & 0.293 & 25.86 & 71.7 & 5.67 \\
\hline & $2048^{2}$ & 0.293 & 81.02 & 104 & 5.73 \\
\hline \multirow[b]{2}{*}{ Algorithm } & & $m=1$ & & & \\
\hline & Size & Err & $\mathrm{Sec}$ & $\begin{array}{c}\text { SpUp } \\
\text { (Par) }\end{array}$ & $\begin{array}{l}\text { SpUp } \\
\text { (Alg) }\end{array}$ \\
\hline \multirow{4}{*}{$\begin{array}{l}\text { RL } \\
\text { It }=1461\end{array}$} & $256^{2}$ & 0.311 & 114.9 & - & - \\
\hline & $512^{2}$ & 0.307 & 644.3 & - & - \\
\hline & $1024^{2}$ & 0.306 & 2574 & - & - \\
\hline & $2048^{2}$ & 0.306 & 11689 & - & - \\
\hline \multirow{4}{*}{$\begin{array}{l}\text { RL_CUDA } \\
\text { It }=1461\end{array}$} & $256^{2}$ & 0.311 & 5.375 & 21.4 & - \\
\hline & $512^{2}$ & 0.307 & 9.656 & 66.7 & - \\
\hline & $1024^{2}$ & 0.306 & 22.41 & 115 & - \\
\hline & $2048^{2}$ & 0.306 & 68.44 & 171 & - \\
\hline \multirow{4}{*}{$\begin{array}{l}\text { SGP } \\
\text { It }=38\end{array}$} & $256^{2}$ & 0.311 & 3.672 & - & 31.3 \\
\hline & $512^{2}$ & 0.308 & 20.36 & - & 31.6 \\
\hline & $1024^{2}$ & 0.307 & 78.20 & - & 32.9 \\
\hline & $2048^{2}$ & 0.306 & 354.0 & - & 33.0 \\
\hline \multirow{4}{*}{$\begin{array}{l}\text { SGP_CUDA } \\
\text { It }=38\end{array}$} & $256^{2}$ & 0.311 & 0.266 & 13.8 & 20.2 \\
\hline & $512^{2}$ & 0.307 & 0.531 & 38.3 & 18.2 \\
\hline & $1024^{2}$ & 0.307 & 1.344 & 58.2 & 16.7 \\
\hline & $2048^{2}$ & 0.306 & 4.188 & 84.5 & 16.3 \\
\hline
\end{tabular}
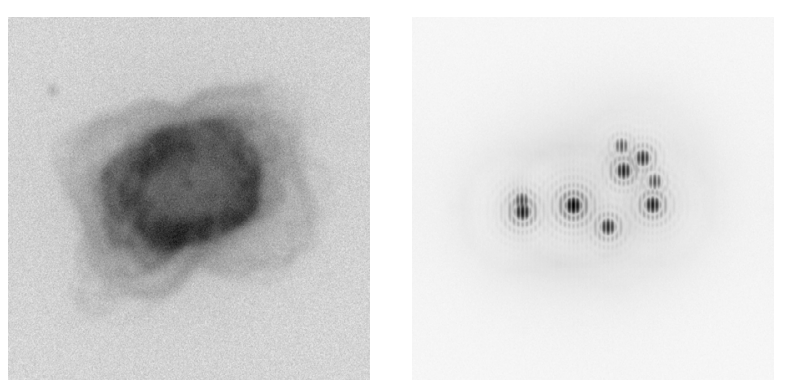

Fig. 5. Interferometric images (horizontal baseline) of the $512 \times$ 512 Nebula with $m=15$ (left panel) and of the star cluster (right panel).

background of about $13.5 \mathrm{mag} \operatorname{arcsec}^{-2}$, corresponding to observations in $K$-band, and with both Poisson and Gaussian noises $\left(\sigma=10 \mathrm{e}^{-} / \mathrm{px}\right)$. In Fig. 5, we show one interferometric image of the nebula, with magnitude 15 , and one interferometric image of the star cluster, both with a horizontal baseline.
Table 5. Reconstruction of the nebula using three equispaced $512 \times$ 512 images.

\begin{tabular}{lcccc}
\hline \hline \multicolumn{5}{c}{$m=10$} \\
Algorithm & It & Err & Sec & SpUp \\
\hline RL & 3401 & 0.032 & 4364 & - \\
RL_CUDA & 3401 & 0.032 & 48.00 & 90.9 \\
OSEM & 1133 & 0.032 & 1602 & - \\
OSEM_CUDA & 1133 & 0.032 & 18.59 & 86.2 \\
SGP & 144 & 0.033 & 220.7 & - \\
SGP_CUDA & 144 & 0.033 & 3.563 & 61.9 \\
\hline \multicolumn{5}{c}{$m=15$} \\
Algorithm & $\mathrm{It}$ & Err & Sec & SpUp \\
\hline RL & 353 & 0.091 & 441.5 & - \\
RL_CUDA & 353 & 0.091 & 4.937 & 89.4 \\
OSEM & 117 & 0.091 & 165.7 & - \\
OSEM_CUDA & 117 & 0.091 & 2.062 & 80.4 \\
SGP & 16 & 0.087 & 26.14 & - \\
SGP_CUDA & 16 & 0.087 & 0.546 & 47.9 \\
\hline
\end{tabular}

\subsubsection{Diffuse objects}

We now provide the results obtained in the case of the nebula with two magnitudes, 10 and 15 . The stopping rule is given again by the minimum rms error. We first consider deconvolution without correction for edge artifacts because the object is within the image domain. The results are reported in Table 5. If we compare the behaviors of single image and multiple image RL, we find that in the second case a larger number of iterations is required, owing to the difficulty in combining the resolutions of the different images to get a unique high-resolution reconstruction. Moreover, the greater cost per iteration has two causes: the first is that the size is $256 \times 256$ in the single case and $512 \times 512$ in the multiple image case; the second is that one single image iteration requires 4 FFTs, while one multiple image iteration, with three images, requires 10 FFTs.

The results confirm that the speedup provided by OSEM with respect to multiple $\mathrm{RL}$ is about 2.5 with a reduction by a factor 3 in the number of iterations (see Sect. 2.3), although the speedup provided by SGP with respect to OSEM of a factor between 6 and 7 is interesting. This speedup presumably decreases as the number of images increases, but a speedup of about 20 is provided by OSEM in the case of 26 images, a number that presumably will never be achieved in the case of LN. Therefore, one can conclude that SGP can be recommended for the deconvolution of LN images. Our CUDA implementations provide an additional speedup of about 80/90 for RL and OSEM, while smaller factors are observed for SGP.

When testing the accuracy of the deconvolution methods with boundary effect correction, we follow the same procedure used in the single image case, i.e. the images are partitioned into four partially overlapping subimages, the methods with boundary effect correction are applied and the final reconstruction is obtained as a mosaic of the four partial reconstructions. The results are reported in Table 6 and confirm the results obtained in the single image case.

\subsubsection{Point-wise objects}

In this case, iterations are pushed to convergence and therefore the stopping rule is given by the condition in Eq. (24); we use different values of tol, specifically $10^{-3}, 10^{-5}$, and $10^{-7}$. In order 
Table 6. Reconstruction of the nebula as a mosaic of four reconstructed subimages with boundary effect correction, also in the case of three equispaced images.

\begin{tabular}{lcccc}
\hline \hline \multicolumn{5}{c}{$m=10$} \\
Algorithm & It & Err & Sec & SpUp \\
\hline RL & 2899 & 0.034 & 13978 & - \\
RL_CUDA & 2899 & 0.034 & 174.2 & 80.2 \\
OSEM & 950 & 0.034 & 5447 & - \\
OSEM_CUDA & 950 & 0.034 & 64.03 & 85.1 \\
SGP & 160 & 0.034 & 873.3 & - \\
SGP_CUDA & 160 & 0.034 & 15.45 & 56.5 \\
\hline \multicolumn{5}{c}{$m=15$} \\
Algorithm & It & Err & Sec & SpUp \\
\hline RL & 243 & 0.094 & 1174 & - \\
RL_CUDA & 243 & 0.094 & 15.28 & 76.8 \\
OSEM & 81 & 0.094 & 479.1 & - \\
OSEM_CUDA & 81 & 0.094 & 5.939 & 80.7 \\
SGP & 11 & 0.087 & 69.88 & - \\
SGP_CUDA & 11 & 0.086 & 1.532 & 45.6 \\
\hline
\end{tabular}

to measure the quality of the reconstruction, we introduce an average relative error of the magnitudes defined by

av_rel_er $=\frac{1}{q} \sum_{j=1}^{q} \frac{\left|m_{j}-\widetilde{m}_{j}\right|}{\widetilde{m}_{j}}$,

where $q$ is the number of stars (in our case $q=9$ ) and $\widetilde{m}_{j}$ and $m_{j}$ are respectively the true and the reconstructed magnitudes. The results are reported in Table 7.

We first point out that, as in the previous cases, we constrain the parallel codes to perform the same number of iterations as the serial ones. This constraint is introduced because the FFT does not have the same precision in the two cases, as already discussed. As a result, the two implementations of the same algorithm do not provide the same error for the same number of iterations. This effect presumably will be removed when a double-precision FFT becomes available for GPU.

Next, we find, as expected, that the number of iterations increases with decreasing values of tol. However, the increase in computation time is not compensated by a significant decrease in the accuracy of the reconstructed magnitudes. For $t o l=10^{-3}$, the accuracy of the estimated magnitudes might already be satisfactory. We observe, however, that with this milder tolerance the accuracy provided by the three algorithms is not the same. Multiple RL and OSEM seem to be slightly more accurate. The accuracy of all algorithms is essentially the same for the smaller tolerances.

As a final experiment, we consider the reconstruction of a binary with high dynamic range (Bertero et al. 2011). It consists of a primary with $m_{1}=10$ (denoted as $S_{1}$ ) and a secondary with $m_{2}=20$ (denoted as $S_{2}$ ). The distance between the two stars is 45 mas (i.e. 9 pixels for the LINC-NIRVANA detector) and the axis of the binary forms an angle of $23^{\circ}$ with the direction of the baseline of the first image. Three equispaced images are generated as in the case of the star cluster, using the same PSFs and the same background.

In this experiment, we need a very small tolerance, i.e. tol $=10^{-7}$, in order to allow SGP to detect the faint secondary. The reason is presumably that SGP requires a projection onto the non-negative orthant, and the existence of this projection can make degrade the appearance of the secondary. In all cases, the results reported in Table 8 are interesting and demonstrate that the magnitude of the secondary can also be estimated with a sufficient accuracy in a reasonable computation time.
Table 7. Reconstruction of the star cluster with three $512 \times 512$ equispaced images.

\begin{tabular}{lcccc}
\hline \hline \multicolumn{5}{c}{$t o l=1 \mathrm{e}-3$} \\
Algorithm & It & Err & Sec & SpUp \\
\hline RL & 319 & $2.39 \mathrm{e}-4$ & 393.4 & - \\
RL_CUDA & 319 & $2.38 \mathrm{e}-4$ & 4.641 & 84.8 \\
OSEM & 151 & $1.63 \mathrm{e}-4$ & 220.8 & - \\
OSEM_CUDA & 151 & $1.62 \mathrm{e}-4$ & 2.421 & 91.2 \\
SGP & 71 & $1.35 \mathrm{e}-3$ & 97.80 & - \\
SGP_CUDA & 71 & $1.29 \mathrm{e}-3$ & 1.641 & 59.6 \\
\hline \multicolumn{5}{c}{ tol $=1 \mathrm{e}-5$} \\
Algorithm & $\mathrm{It}$ & Err & Sec & SpUp \\
\hline RL & 1385 & $6.65 \mathrm{e}-5$ & 1703 & - \\
RL_CUDA & 1385 & $6.64 \mathrm{e}-5$ & 19.38 & 87.9 \\
OSEM & 675 & $5.64 \mathrm{e}-5$ & 980.6 & - \\
OSEM_CUDA & 675 & $5.64 \mathrm{e}-5$ & 10.75 & 91.2 \\
SGP & 337 & $5.89 \mathrm{e}-4$ & 455.2 & - \\
SGP_CUDA & 337 & $1.79 \mathrm{e}-4$ & 7.187 & 63.3 \\
\hline \multicolumn{5}{c}{ tol $=1 \mathrm{e}-7$} \\
Algorithm & $\mathrm{It}$ & Err & Sec & SpUp \\
\hline RL & 7472 & $5.64 \mathrm{e}-5$ & 9180 & - \\
RL_CUDA & 7472 & $5.98 \mathrm{e}-5$ & 104.8 & 87.6 \\
OSEM & 3750 & $6.13 \mathrm{e}-5$ & 5442 & - \\
OSEM_CUDA & 3750 & $5.98 \mathrm{e}-5$ & 59.52 & 91.4 \\
SGP & 572 & $7.37 \mathrm{e}-5$ & 772.6 & - \\
SGP_CUDA & 572 & $7.05 \mathrm{e}-5$ & 12.20 & 63.3 \\
\hline
\end{tabular}

Notes. The error is the average relative error in the magnitudes defined in Eq. (27).

Table 8. Reconstruction of the binary with high dynamic range (image size: $256 \times 256$ ).

\begin{tabular}{cccc}
\hline \hline \multirow{4}{c}{$t o l=1 \mathrm{e}-7$} \\
Algorithm & It & Sec & SpUp \\
\hline RL & 30765 & 6108 & - \\
RL_CUDA & 30765 & 292.9 & 20.9 \\
OSEM & 14291 & 3216 & - \\
OSEM_CUDA & 14291 & 156.0 & 20.6 \\
SGP & 2073 & 482.8 & - \\
SGP_CUDA & 2073 & 28.59 & 16.9 \\
\hline \multirow{4}{*}{ Magnitude } \\
Algorithm & Star & Real & Reconstructed \\
\hline \multirow{2}{*}{ RL } & S1 & 10 & 10.0001 \\
& S2 & 20 & 20.1841 \\
\hline \multirow{2}{*}{ OSEM } & S1 & 10 & 10.0001 \\
& S2 & 20 & 20.0919 \\
\hline \multirow{2}{*}{ SGP } & S1 & 10 & 10.0001 \\
& S2 & 20 & 20.2683 \\
\hline
\end{tabular}

\section{Discussion}

The codes of the algorithms presented and discussed in this paper can be freely downloaded ${ }^{2}$. A MATLAB code of SGP for single image deconvolution is also available at the same URL, which enables one to compare the IDL and MATLAB implementations on one's own computer.

The paper is based on the RL algorithm and its generalizations to boundary effect correction and multiple image deconvolution being scaled gradient methods, where the scaling is provided by the current iterate. Therefore, it is possible to attempt to improve the efficiency of these algorithms in the framework of the SGP approach proposed in Bonettini et al. (2009). As already shown in that paper, the SGP version of RL provides a considerable increase in efficiency.

\footnotetext{
${ }^{2}$ At the URL http://www. unife/prin/software
} 
The results given in the previous section demonstrate that SGP allows a significant speedup of all the RL-type algorithms considered in this paper, even if the speedup depends considerably on the specific object to be reconstructed and, for a given object, on the noise level; it ranges from about 4 in the case of multiple images of the star cluster (Table 7), to more than 30, in the case of a single image of the galaxy (Table 4). A more accurate investigation of the speedup achievable would require application to a broader data set of astronomical objects as well as to images with different noise levels and noise realizations. In all cases, we believe that the results presented in this paper are sufficient to demonstrate that SGP is a valuable acceleration of RL-like algorithms and that in several cases it allows a considerable reduction in computational time.

The speedup provided by GPU implementation is consistent with the results reported in Ruggiero et al. (2010). The speedup of RL-algorithms is greater than that of SGP-algorithms because the main computational kernel of RL is FFT, while SGP is also based on the computation of steplengths, etc. Nevertheless, the gain with respect to RL is very significant, in some cases, allowing us to deconvolve a $2048 \times 2048$ image in a few seconds. We analyzed the use of a multi-thread FFTW in our C implementation of the SGP algorithm, obtaining an improvement in the performance with respect to the corresponding serial code, which is however definitely lower than that achieved with the CUDA version.

We conclude by emphasizing that in this paper we have considered a maximum likelihood approach for the image deblurring problems and used an early stopping of the iterative procedures to mimic a regularization effect. However, the SGP method can also be applied to regularized deconvolution in the framework of a Bayesian approach. The main problem would then be to decide on a rule to determine a suitable scaling for a given regularization function, since the scaling should depend on this function. Such a rule can be provided by the split-gradient method (SGM) proposed by Lantéri et al. (2001, 2002), which can be considered an improvement of the one-step late (OSL) method proposed by Green (1990): the OSL scaling does not always yield positive values, while the SGM scaling does.

The scaling of SGM combined with SGP has been already tested in the case of Poisson denoising (Zanella et al. 2009) and Poisson deblurring (Staglianò et al. 2011), which are both based on edge-preserving regularization. In both cases, this combination leads to very efficient algorithms. The SGM scalings can also be designed for other kinds of regularization, and for a discussion of the case for Poisson data we refer to Bertero et al. (2009, 2011).

Work is in progress to develop a library of SGP algorithms for Poisson data deconvolution with a number of different kinds of regularization.

Acknowledgements. This work has been partially supported by MIUR (Italian Ministry for University and Research), PRIN2008 "Optimization Methods and
Software for Inverse Problems", grant 2008T5KA4L, and by INAF (National Institute for Astrophysics) under the contract TECNO-INAF 2010 "Exploiting the adaptive power: a dedicated free software to optimize and maximize the scientific output of images from present and future adaptive optics facilities".

\section{References}

Adorf, H.-M., Hook, R. N., Lucy, L. B., \& Murtagh, F. D. 1992, in ESO Conf. Workshop Proc., 41, 99

Anconelli, B., Bertero, M., Boccacci, P., \& Carbillet, M. 2005, A\&A, 430, 731 Anconelli, B., Bertero, M., Boccacci, P., Carbillet, M., \& Lanteri H. 2006, A\&A, 448, 1217

Arcidiacono, C., Diolaiti, E., Tordi, M., et al. 2004, Appl. Opt., 43, 4288

Barrett, H. H., \& Meyers, K. J. 2003, Foundations of Image Science (New York: Wiley and Sons), 1047

Barzilai, J., \& Borwein, J. M. 1988, IMA J. Numer. Anal., 8, 141

Benvenuto, F., Zanella, R., Zanni, L., \& Bertero, M. 2010, Inverse Probl., 26, 025004

Bertero, M., \& Boccacci, P. 2000, A\&AS, 144, 181

Bertero, M., \& Boccacci, P. 2005, A\&A, 437, 369

Bertero, M., Boccacci, P., Desiderà, G., \& Vicidomini, G. 2009, Inverse Probl., 25,123006

Bertero, M., Boccacci, P., Talenti, G., Zanella, R., \& Zanni, L. 2010, Inverse Probl., 26, 105004

Bertero, M., Boccacci, P., La Camera, A., Olivieri, C., \& Carbillet, M. 2011, Inverse Probl., 27, 113001

Bertsekas, D. P. 2003, Nonlinear Programming, 2nd edn. (Athena Scientific), 29

Biggs, D. S. C., \& Andrews 1997, Appl. Opt., 36, 1766

Bonettini, S., \& Prato, M. 2010, Inverse Probl., 26, 095001

Bonettini, S., Zanella, R., \& Zanni, L. 2009, Inverse Probl., 25, 015002

Csiszár, I. 1991, Ann. Stat., 19, 2032

Favati, P., Lotti, G., Menchi, O., \& Romani, F. 2010, Inverse Probl., 26, 085013

Frassoldati, G., Zanni, L., \& Zanghirati, G. 2008, J. Indust. Manag. Optim., 4, 299

Green, P. J. 1990, IEEE Trans. Med. Imag., 9, 84

Herbst, T. M., Ragazzoni, R., Andersen, D., et al. 2003, Proc. SPIE, 4838, 456

Hudson, H. M., \& Larkin, R. S. 1994, IEEE Trans. Med. Imag., 13, 601

Iusem, A. N. 1991, Math. Methods Appl. Sci., 14, 573

Lantéri, H., Roche, M., Cuevas, O., \& Aime C. 2001, Signal Processing, 81, 945 Lanterí, H., Roche, M., \& Aime, C. 2002, Inverse Probl., 18, 1397

Llacer, J., \& Núñex, J. 1990, in The restoration of HST images and spectra, ed. R. L. White, \& R. J. Allen, Space telescope Science Institute, 62

Loris, I., Bertero, M., De Mol, C., Zanella, R., \& Zanni, L. 2009, Appl. Computat. Harmon. A, 27, 247

Lucy, L. B. 1974, AJ, 79, 745

Lucy, L. B., \& Hook, R. N. 1992, ASP Conf. Ser., 25, 277

Natterer, F., \& Wübbeling, F. 2001, Mathematical Methods in Image Reconstruction, SIAM, 118

Richardson, W. H. 1972, JOSA, 62, 55

Ruggiero, V., Serafini, T., Zanella, R., \& Zanni, L. 2010, J. Glob. Optim., 48, 145

Serafini, T., Zanghirati, G., \& Zanni, L. 2005, Opt. Meth. Software, 20, 353

Shepp, L. A., \& Vardi, Y. 1982, IEEE Trans. Med. Imag., 1, 113

Snyder, D. 1990, in The restoration of HST images and spectra, ed. R. L. White, \& R. J. Allen, Space telescope Science Institute, 56

Snyder, D. L., Helstrom, C. W., Lanterman, A. D., Faisal, M., \& White, R. L. 1994, JOSA, A12, 272

Staglianò, A., Boccacci, P., \& Bertero, M. 2011, Inverse Probl., 27, 125003

Zanella, R., Boccacci, P., Zanni, L., \& Bertero, M. 2009, Inverse Probl., 25, 045010

Zhou, B., Gao, L., \& Dai, Y. H. 2006, Comput. Optim. Appl., 35, 69 